\title{
Factores de riesgo que influyen en embarazos \\ en adolescentes
}

\author{
Risk factors influencing pregnancy in adolescents
}

Fatores de risco que influenciam a gravidez na adolescência

\author{
Andrea Amizola \\ andrea_amaq@hotmail.com
}

Recibido enero 2019 / Revisión febrero 2019 / Aceptado 1 de mayo 2019

\begin{abstract}
RESUMEN
Introducción: El embarazo en la adolescencia constituye un problema de salud en el mundo debido a las múltiples complicaciones tanto para la madre como para el niño además de las implicaciones sociales como perpetuación del ciclo de la pobreza, falta de oportunidades educativas, ocupacionales y socio-económicos. Objetivo: Identificar los factores de riesgo que influyen embarazos en adolescentes de 15 a 19 años de la Unidad Educativa Viliroco "B" del municipio de Viacha, Provincia Ingavi, durante el cuarto trimestre de la gestión. Materiales y métodos: Estudio descriptivo, transversal, prospectivo y observacional usando como método de recolección de datos encuestas realizadas a la población adolescente entre 14 y 19 años. Resultados y Discusión: La falta de información representa el $37 \%$, también se pudo evidenciar que el $82 \%$ de las adolescentes si viven con sus padres y $18 \%$ no viven con sus padres, el $45 \%$ de la encuestadas ya iniciaron con la vida sexual de la cuales el $20 \%$ iniciaron con la vida sexual a los 14 años, el $15 \%$ fueron orientados por su madre y el $45 \%$ por el personal de salud. Conclusión: El conocimiento en las adolescentes de 15 a 19 años sobre sexualidad es poco, los factores de riesgo asociados a embarazos en adolescentes fueron la falta de información, seguido el alcoholismo y la edad promedio de inicio a la vida sexual la cual fue de 14 años.
\end{abstract}

Palabras clave: embarazo; embarazo en adolescencia; adolescente

\section{ABSTRACT}

Introduction: Pregnancy in adolescence constitutes a health problem in the world due to the multiple complications for both mother and child, in addition to the social implications such as perpetuation of the cycle of poverty, lack of educational, occupational and social opportunities. Economic. Objective: To identify the risk factors that influence pregnancies in adolescents between 15 and 19 years of age from the Viliroco "B" Educational Unit of the municipality of Viacha, Ingavi Province, during the fourth quarter of the administration. Materials and methods: Descriptive, cross-sectional, prospective and observational study using surveys of the adolescent population between 14 and 19 years as a data collection method. Results and Discussion: The lack of information represents $37 \%$, it was also evident that $82 \%$ of adolescents if they live with their parents and $18 \%$ do not live with their parents, $45 \%$ of the respondents already started with sexual life of which $20 \%$ started with sexual life at age $14,15 \%$ were guided by their mother and $45 \%$ by health personnel. Conclusion: Knowledge of sexuality in adolescents between 15 and 19 years old is low, the risk factors associated with teenage pregnancies were the lack of information, followed by alcoholism and the average age at the beginning of sexual life, which was 14 years.

Key words: pregnancy; teenage pregnancy; Teen
AA: Universidad Mayor de San Andrés, Bolivia 
AA: Universidad Mayor de San Andrés, Bolivia

\section{RESUMO}

Introdução: A gravidez na adolescência constitui um problema de saúde no mundo devido às múltiplas complicações para mãe e filho, além de implicações sociais como a perpetuação do ciclo de pobreza, falta de oportunidades educacionais, ocupacionais e sociais. econômico. Objetivo: Identificar os fatores de risco que influenciam a gravidez em adolescentes entre 15 e 19 anos da Unidade Educacional Viliroco "B" do município de Viacha, província de Ingavi, durante o quarto trimestre da administração. Materiais e métodos: Estudo descritivo, transversal, prospectivo e observacional, utilizando como método de coleta de dados a população adolescente entre 14 e 19 anos. Resultados e Discussão: A falta de informação representa $37 \%$, também ficou evidente que $82 \%$ dos adolescentes se moram com os pais e $18 \%$ não moram com os pais, $45 \%$ dos entrevistados já iniciaram a vida sexual dos quais $20 \%$ iniciaram a vida sexual aos 14 anos, $15 \%$ foram orientados pela mãe e $45 \%$ pelo pessoal de saúde. Conclusão: $O$ conhecimento da sexualidade em adolescentes entre 15 e 19 anos é baixo, os fatores de risco associados à gravidez na adolescência foram a falta de informação, seguida pelo alcoolismo e a idade média no início da vida sexual, 14 anos.

Palavras-chave: gravidez; gravidez na adolescência; adolescente

\section{INTRODUCCIÓN}

a Organización Mundial de la Salud

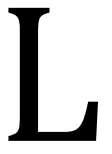
(OMS) define la adolescencia como la etapa que transcurre entre los 11 y 19 años, considerándose dos fases: la adolescencia temprana 11 a 14 años y la adolescencia tardía 15 a 19 años (1). Los jóvenes de la actualidad, se enfrentan a un problema: la sociedad y grupos sociales en los que conviven, los cuales conllevan a la toma de decisiones que repercuten en su juventud, arrastrándolos al uso de drogas, pandillerismo y embarazo en adolescentes.

La tasa de adolescentes entre 15 y 19 años embarazadas en América Latina y el Caribe, es solo superada por África. El 20\% de la región, corresponde a mujeres menores de 20 años, 4,2 millones de abortos inseguros se practican en la región cada año (2).

Los riesgos del embarazo en la adolescencia, se incrementan en los países en vías de desarrollo $(3,4)$. Algunos organismos internacionales como la UNICEF, señala que entre el 20 y el $46 \%$ de las mujeres, en la mayoría de un grupo seleccionado de países en vías de desarrollo, tienen su primer hijo antes de los 18 años de edad y al llegar a los 20 años, del 30 al 67\% han tenido su primer parto. En los Estados Unidos de Norteamérica, esto ocurre en el $8 \%$ de la población adolescente (5).

Bolivia, es uno de los países con mayor índice de embarazo adolescente, pues comparte con Venezuela los primeros puestos en la lista de Sudamérica. El Fondo de Población de las Naciones Unidas (UNFPA), a través de numerosos estudios, ha calculado que la tasa de embarazos en la adolescencia en Bolivia llega al 21\%, muy superior al promedio latinoamericano que estaría en 18\%. Los embarazos registrados en todo el país anualmente, están entre 250 mil y 300 mil, y de ellos 60.000 corresponden a adolescentes cuyas edades están comprendidas entre 15 y 19 años (6).

En Bolivia la población adolescente de 10 a 19 años, representa alrededor del $23 \%$ de la población total del país, un $49 \%$ corresponde a adolescentes de sexo femenino, en quienes se estima, según datos de la Encuesta Nacional de Salud (ENDSA 2008), que cerca de un $18 \%$ ya son madres o están embarazadas (de 15 a 19 años). Al menos, la mitad de estos embarazos no fueron planificados al momento de la concepción (6). 
Según ENDSA 2008, los municipios con mayores tasas de embarazo en adolescentes son: El Alto que registra un promedio de 83\%, Santa Cruz 71\%, Guayaramerín 76\%, Riberalta 71\% y Colcapirhua $74 \%$. La misma fuente revela que de las adolescentes encuestadas que ya iniciaron relaciones sexuales, un $60 \%$ habían estado embarazadas en algún momento y resulta llamativo que el 9\% corresponde a adolescentes menores de 15 años. En los Municipios de la Paz, el 40\% de los 3.800 embarazos en adolescentes del departamento, se registró en municipios atendidos por cinco redes de salud $(6,9)$.

El embarazo en la adolescencia, como problema de salud, tiene factores de riesgo estudiados y agrupados en relación a sus características multifactoriales. Esta investigación tiene como objetivo, identificar los factores de riesgo que influyen embarazos en adolescentes de 15 a 19 años de la Unidad Educativa Viliroco "B" del municipio de Viacha, Provincia Ingavi durante el cuarto trimestre de la gestión.

\section{MATERIALES Y MÉTODOS}

0 e realizó un estudio de tipo descriptivo, transversal, prospectivo y observacional, se usó como método de recolección de datos la encuesta, realizada a la población adolescente entre 14 y 19 años de la Unidad Educativa Viliroco "B" del municipio de Viacha, durante el cuarto trimestre de la gestión. Esta permitió medir factores de riesgo, dentro de la población objeto de estudio. La población estuvo constituida por 110 adolescentes, se trabajó con la totalidad de la muestra al $100 \%$ así que no hubo muestra. La investigación se rige por los principios éticos internacionales en materia investigativa. Se informó a los padres, representantes y adolescentes sobre los alcances del estudio y se solicitó el consentimiento informado. La investigación fue aprobada por el Comité de Ética.

\section{RESULTADOS Y DISCUSIÓN}

$\mathrm{U}$

na vez tabulados los resultados, se presenta detalladamente el análisis correspondiente:

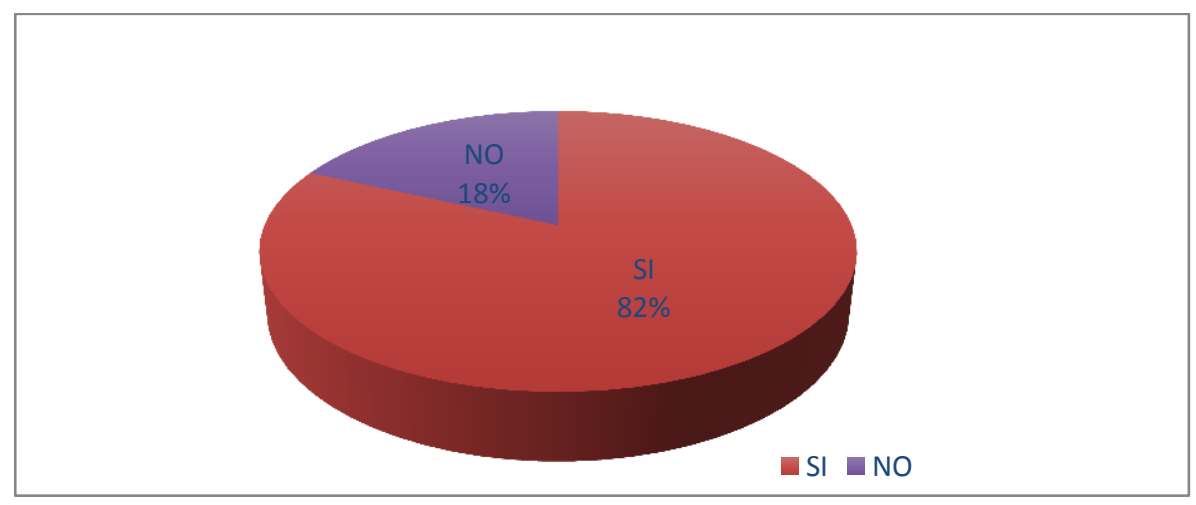

Gráfico 1. Adolescentes que conviven con sus padres

De la muestra de 110 adolescentes de 15 a 19 años encuestadas, el $82 \%$ viven con sus padres, mientras que las que no viven con sus padres, representan el $18 \%$ de la población adolescente considerada. 


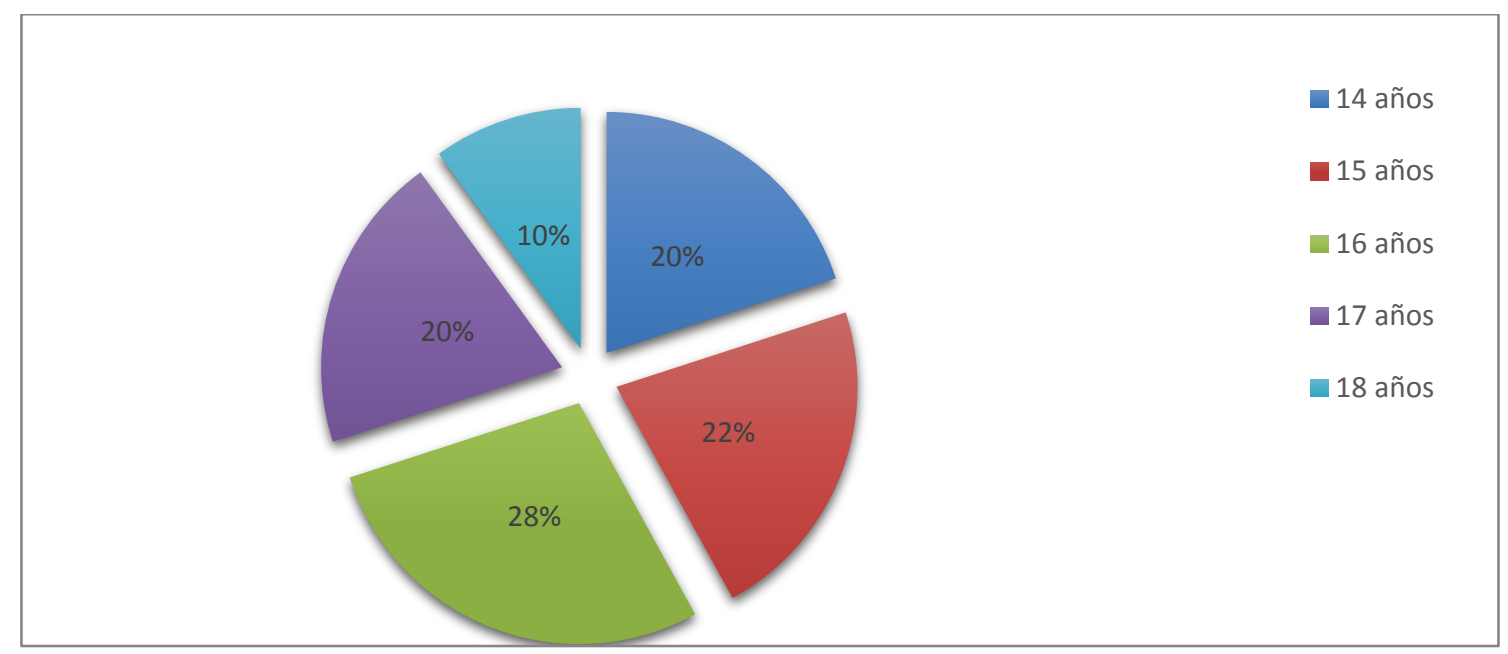

Gráfico 2. Edad de inicio de relaciones sexuales en los adolescentes

$28 \%$ de estas adolescentes entre 14 y 19 años encuestados, iniciaron relaciones sexuales a los 16 años de edad, seguidas por un $22 \%$ de las adolescentes quienes iniciaron relaciones sexuales a los 15 años. Tanto las adolescentes de 17 años como las de 14 años representaron el $20 \%$ de la población, mientras que solo un $10 \%$ de ellas, iniciaron sus relaciones sexuales a los 18 años, cuando ya están más preparadas fisiológica, emocional y mentalmente para el embarazado y son más responsables por sus actos.

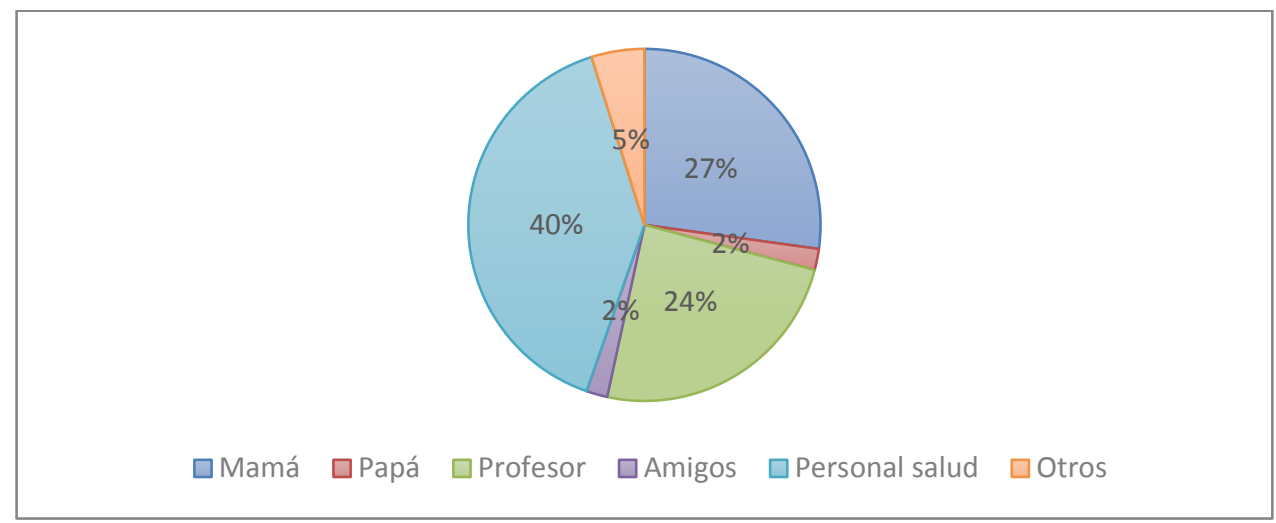

Gráfico 3. Educación y orientación sexual recibida por los adolescentes

Con respecto a recibir educación y orientación sexual y de quién provino ésta, el $40 \%$ de las adolescentes encuestadas, recibió orientación sexual de algún personal de salud, el $27 \%$ mencionó haber recibido orientación sexual de su madre, el $24 \%$ de las adolescentes señaló que fue impartida por profesores, solo un 5\% fue recibida de otro tipo de personas y $2 \%$ señalaron haber recibido orientación sexual de amigos y padres. 


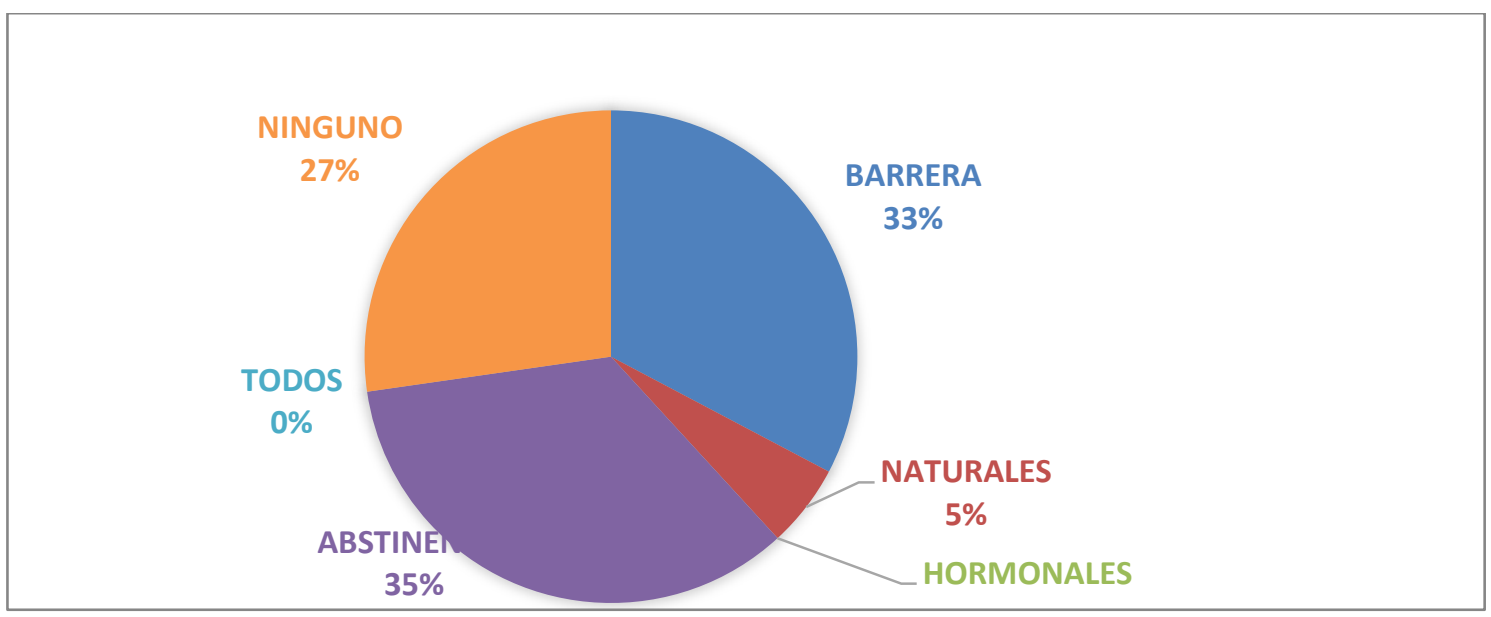

Gráfico 4. Conocimiento de métodos anticonceptivos en los adolescentes

Conocen la abstinencia como método anticonceptivo $35 \%$ de las encuestadas, 33\% conocen el método de barrera, $27 \%$ de las adolescentes entre 14 y 19 años encuestados, señaló no conocer ningún tipo de método anticonceptivo, mientras que solo un 5\%, mencionó conocer los métodos naturales de anticoncepción.

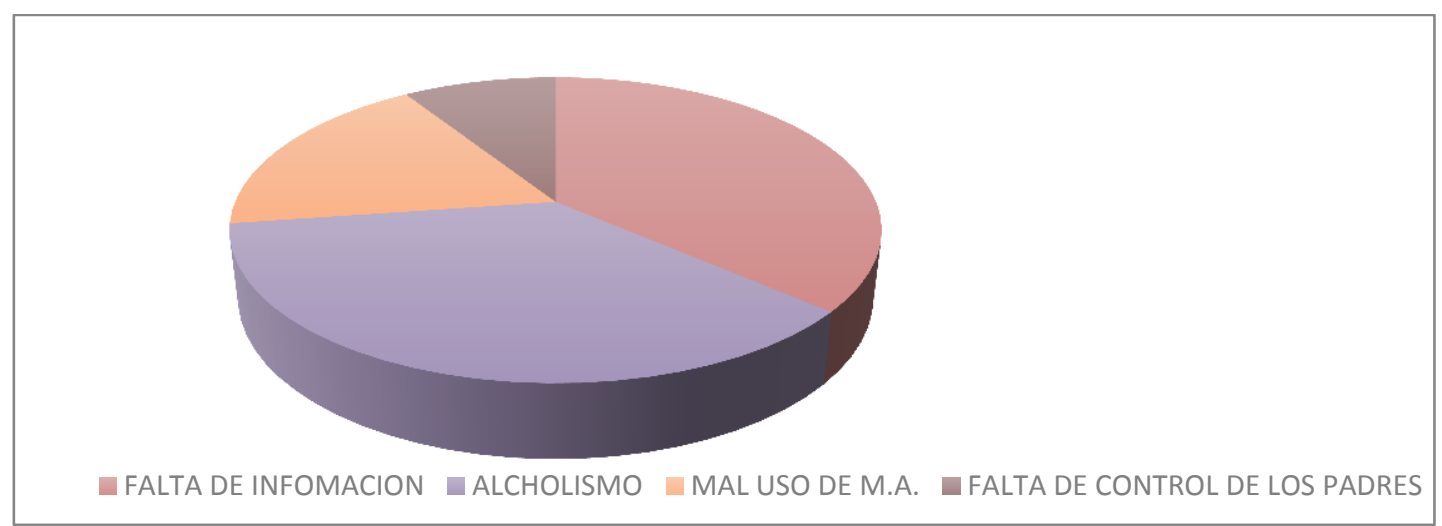

Gráfico 5. Factores de riesgos para el embarazo en adolescentes

Diversas razones expresan las adolescentes, en cuanto a los factores de riesgo para un embarazo y que la hacen propensa a un embarazo, el mayor porcentaje falta de información.

\section{Discusión}

En el presente estudio se encontró en las adolescentes, un inicio precoz de relaciones sexuales a partir de los 14 años de edad, dato que coincide con la investigación Casanueva et al (7) realizada en adolescentes mexicanas. Las relaciones sexuales en comunidades como la estudiada, se caracterizan por ser precoces, sin estabilidad de la pareja, con mayor riesgo biológico y social del embarazo.

En este trabajo se encontró que el mayor factor de riesgo, es la falta de información. Al respecto señaló Llanes (8), que el bajo nivel educativo y falta de información influyen en el embarazo precoz. Cuando existe un proyecto de vida que prioriza alcanzar un determinado nivel educativo y posponer la maternidad para la edad adulta, es más probable que los jóvenes, aun teniendo relaciones sexuales, adopten una prevención efectiva del embarazo. 
Muchos de los adolescentes pueden no ser conscientes de los riesgos que implica ser activos sexualmente. Por tal motivo, este grupo etario necesita servicios de Salud Sexual Reproductivos (SSR) para que reciban información sobre las formas de evitar embarazo no deseado y cómo disminuir los riesgos de adquirir una Infección de Transmisión Sexual (8).

El hecho de que la mayoría de las adolescentes estudiadas vivan con sus padres es una fortaleza, es asociado en forma protectora del embarazo adolescente: una tardía iniciación en la vida sexual, el énfasis en la abstinencia en la educación sexual, la actitud firme, apoyo y comunicación de los padres $(9,10)$.

Entre las causas del embarazo en la adolescencia, se encuentran el matrimonio a edades tempranas y el rol de género que tradicionalmente se asigna a la mujer; la práctica de las relaciones sexuales de los jóvenes sin métodos anticonceptivos; la presión de los compañeros, que alientan a los adolescentes a tener relaciones sexuales; el consumo de bebidas alcohólicas y otras drogas que producen una reducción en la inhibición, que puede estimular la actividad sexual no deseada; carecer de información y conocimientos suficientes sobre los métodos anticonceptivos y el no tener un fácil acceso a su adquisición y la falta de una buena educación sexual.

La incidencia de embarazos es mayor en adolescentes de escasos recursos económicos, residentes en las regiones más pobres, emigrantes de zonas rurales y en poblaciones menos. Las adolescentes embarazadas son privadas del desarrollo por disminuir educadas sus capacidades para la educación (11).

Un embarazo en la adolescencia implica repercusiones biológicas, sociales, económicas y psicológicas que clasifican la situación como de muy alto riesgo. $(11,12)$.

\section{CONCLUSIÓN}

$\mathrm{E}$ l inicio temprano de las relaciones sexuales así como la falta de información constituyen factores de riesgo para embarazo en las adolescentes de la Unidad Educativa Viliroco "B". La orientación sexual recibida por el grupo, no ha contribuido a aumentar el caudal de información relativa a los métodos anticonceptivos que le permitieran hacer uso de ellos para evitar el embarazo precoz. Se hace necesario desde una visión integral, involucrar a la familia, la sociedad, los medios de comunicación, el sector salud y educativo, entre otros, con estrategias pedagógicas y un abordaje amplio del tema, para influir favorablemente en la población adolescente acerca de la sexualidad responsable.

- Conflicto de intereses: Ninguno declarado por el autor.

- Financiación: Autofinanciado.

- Agradecimientos: Ninguno declarado por el autor.

\section{REFERENCIAS BIBLIOGRÁFICAS}

1. OMS. El embarazo en la adolescencia. Disponible en: https://www.who.int > topics > maternal > adolescent_pregnancy. [Consultado 201823 de febrero]

2. Quintero A, Rojas H. El embarazo a temprana edad, un análisis desde la perspectiva de madres adolescentes. Revista Virtual Universidad Católica del Norte. [Internet].2015; 44(2): 222$237 . \quad$ Disponible en: http://www.redalyc.org/articulo.oa? id $=194238608016$

3. Manrique R., Rivero A., Ortunio M., Rivas M., Cardozo R., Guevara H. Parto Pretérmino en adolescentes. Rev Obstet Ginecol Venez [Internet].2008; 68(3):141-143. Disponible en: https://www.researchgate.net , publication > 262551626_Parto_ Pretermino 
4. Ministerio de Salud. Coordinación Nacional de Salud Sexual y Reproductiva. Guías de manejo de las complicaciones en el embarazo. Panamá, 2009. Disponible

en http://www.minsa.gob.pa/sites/default /files/programas/guias-complicacionesembarazo_diciembre_2015.pdf

5. United Nations Children's Fund. UNICEF. Programming for safe motherhood. Headquarters. Health. Programme division 2005. Disponible en https://www.unicef.org/about/execboa rd/files/UNICEF_Programming_for_Chil dren_-

_Orientation_2018_Board_Members_Jan_ 201....pdf

6. Ministerio de Salud del Estado Plurinacional de Bolivia. Salud: embarazo en adolescente. La Paz. 2017. [Consultado 201822 de mayo]. Disponible en https://consejoderedaccion.org/webs /opendata/docs/PLAN\%20PLURINAC IONAL\%20PREVENCION\%20EMBAR AZO\%20ADOLESCENTE\%20(3).pdf

7. Casanueva E, Jiménez J, MezaCamacho, C, Mares M, Simon L. Prevalence of nutritional deficiencies in Mexican adolescent women with early and late prenatal care. Arch Latinoam Nutr 2003[citado 201822 de enero]; (1):35-8

8. Llanes A, Quevedo N. Embarazo en la adolescencia. Intervención educativa.
AMC [Internet]. 2009 [citado 201822 de enero]; 13(1). Disponible en: http://scielo.sld.cu/scielo.php?script= sci_arttext\&pid=S1025025009000100009\&

9. López Y. Embarazo en la adolescencia y su repercusión biopsicosocial sobre el organismo de la madre y de su futuro hijo. Rev. Cubana Enfermer [Internet]. 2012 Mar [citado 2018 enero]; 28(1): 23-36. Disponible en: http://scielo.sld.cu/scielo.php?script= sci_arttext\&pid=S0864-

03192012000100004

10. Sadia N. Los jóvenes, en situación de riesgo y necesitados de información y servicios. New York: Fondo de Población de las Naciones Unidas; 2006

11. Rivera J. Juventudes en América Latina: una reflexión desde la experiencia de la exclusión y la cultura. Pap. poblac [Internet]. 2013[citado 201822 de enero]; 19(75); 9-34. Disponible en: http://www.scielo.org.mx/scielo.php? script=sci_arttext\&pid=S1405-

12. De la Peña-Fernández $M$. Conducta antisocial en adolescentes factores de riesgo y protección. Tesis de Grado. Facultad de Psicología. Universidad Complutense de Madrid. 2010. Disponible en https://eprints.ucm.es/12024/1/T28 264.pdf 